\title{
Comportamiento organizacional en la gestión educativa 2020
}

\section{RESUMEN}

Gilber Macedo Vásquez gmav1967@gmail.com

Escuela de posgrado

Universidad César Vallejo

ORCID: 0000-0002-0727-128X

José Manuel Delgado Bardales jmdelgadob@ucvvirtual.edu.pe Escuela de posgrado

Universidad César Vallejo ORCID:0000-0001-6574-2759

Scopus autor ID: 24070333700

Código Renacyt: P0050554

La investigación tuvo como objetivo Caracterizar el comportamiento organizacional en la mejora de la gestión educativa. El estudio fue básico de diseño descriptivo con revisión sistemática, las revisiones sistemáticas son investigaciones científicas en las cuales la unidad de análisis son los estudios originales primarios, constituyen una herramienta esencial para sintetizar la información científica disponible. De los 10 artículos científicos, sobre el comportamiento organizacional en la gestión educativa en el contexto de la crisis económica internacional, corresponden a diferentes bases de datos, todos ellos al tipo y diseño de estudios descriptivos. El comportamiento organizacional asegura un mejor desarrollo de la gestión educativa, la organización facilita procesos de desarrollo institucional que responde a los objetivos estratégicos. El comportamiento organizacional en la gestión educativa es deficiente dado que los usuarios internos y externos no se sienten satisfechos con el servicio brindado por parte los trabajadores direccionados por el líder de las Instituciones Educativas.

Existe un proceso de innovación institucional para fortalecer la gestión intelectual asimismo se cuenta con personal especializado en la gestión del conocimiento

Palabras clave: Recaudación, tributaria, municipal. 


\title{
Organizational behavior in educational management 2020
}

\begin{abstract}
The research aimed to characterize the organizational behavior in the improvement of educational management. The study had a basic descriptive design with systematic review, systematic reviews are scientific investigations in which the unit of analysis are the original primary studies, they constitute an essential tool to synthesize the available scientific information. Of the 10 scientific articles on organizational behavior in educational management in the context of the international economic crisis, they correspond to different databases, all of them to the type and design of descriptive studies. Organizational behavior ensures better development of educational management, the organization facilitates institutional development processes that respond to strategic objectives Organizational behavior in educational management is deficient given that internal and external users do not feel satisfied with the service provided by the workers directed by the leader of the Educational Institutions. There is a process of institutional innovation to strengthen intellectual management, as well as specialized personnel in knowledge management
\end{abstract}

Keywords: Collection, tax, municipal.

Artículo recibido: 03 nov. 2020 Aceptado para publicación: 07 dic. 2020

Correspondencia gmav1967@gmail.com Conflictos de Interés: Ninguna que declarar 


\section{INTRODUCCIÓN}

Las instituciones públicas como las escuelas técnicas, Institutos Superiores o Universidades deben dar cuenta de un proceso de calidad educativa y a la vez de una formación humana en valores que reviertan el mensaje social individualista para los alumnos y sus familias. Lo que podemos ofrecer es una respuesta organizativa tal, que permita abrir la institución a la cotidianeidad del niño, del adolescente y del adulto, que sea capaz de mantener puertas adentro, un clima ordenado de pertenencia, favorable y valorativa para la formación académica del estudiante, dimanada de la armonía institucional y el comportamiento organizacional. (Gnanarajan; Ariyaratnam; Kengatharan; Navaneethakrishnan; Velnampy, Thirunavukkarasu, 2014). En ese contexto, es necesario socializar con la comunidad educativa los resultados, a fin de contrastar con lo que ocurre desde el "afuera" y el "adentro" del propio sistema, para que reflexionemos juntos en términos de un comportamiento organizacional acorde con la misión institucional.

Para ello, en primer término, se debe identificar y los rasgos comunes que tienen hoy las instituciones educativas, escuelas técnicas, Institutos Superiores o Universidades. En efecto, el enfoque sistémico de nuestras instituciones educativas permite abordar sus límites, el rol institucional que corresponde a las jerarquías funcionales y su relación con las otras instancias o niveles educativos. Es importante, además, saber que el responsable de la gestión escolar ejerza liderazgo y conozca los aspectos comunicacionales derivados de su relación con el entorno interno y con otras dimensiones transorganizacionales, ya que estas interacciones son a menudo causales de conflictos en el seno de las instituciones educativas. (Munir; Yasin; Shabbir; Ali; Tariq; Chani; Orangzab, Muhammad; Abbas, Mazhar; 2019 Special Issue, Vol. 6, p1-21, 21p)

No obstante, los directivos quieren estar seguros de que sus organizaciones podrán soportar mucho tiempo y para ello, en nuestros tiempos, se hace indispensable conocer sobre el comportamiento humano en las organizaciones y éste será entendible sólo cuando lo analizamos de manera holística, sistémica, multidisciplinaria e interdisciplinaria y en donde las relaciones personas - organización deben verse como un todo, teniéndose como entendido que las habilidades técnicas son necesarias para el éxito en la gestión escolar. (Martín; Leiva; Brenes; 2019, Vol. 64 Issue 2, p1-18. 18p.). 
El recurso humano es un ámbito totalmente sensible dentro de las organizaciones, el más importante sin duda alguna, que depende en gran medida de la cultura existente en cada organización. Por lo que, al encontrarnos ante constantes cambios y diversificación tecnológica, es importante encausar la presente investigación respecto a la problemática que se presenta en las instituciones educativas, en relación al impacto que tiene la motivación en el comportamiento de los individuos, lo que se refleja en su satisfacción y desempeño laboral. En todos los ámbitos de la existencia humana interviene la motivación como mecanismo para lograr determinados objetivos y alcanzar determinadas metas; ya que representa un fenómeno humano universal de gran trascendencia para los individuos y la sociedad. Muchas organizaciones, e instituciones estatales se quejan del rendimiento laboral de sus trabajadores, aluden que no se identifican con su centro de labores, cambian de personal, al darse cuenta que sus trabajadores realizan un trabajo deficiente que perjudica el clima institucional, el servicio, la productividad y las finanzas de la organización. (Chiavenato, 2007, p. 441).

Por su parte los trabajadores o empleados se quejan de sus superiores y expresan que los ven únicamente jefes, supervisores que sólo buscan figurar ante la institución y no como el verdadero potencial que ellos significan con sentimientos, emociones y necesidades de reconocimiento. Por eso, para comprender el comportamiento humano es fundamental conocer la motivación humana; entendida como el impulso que lleva a la persona a actuar de determinada manera, es decir, que da origen a un comportamiento específico. En ese sentido, existe un problema entre empleador y empleado, que se debe a una variedad de factores, uno de ellos es la falta de motivación y reconocimiento que no se le da al potencial humano de la organización. (Arredondo Trapero, Florina; Vázquez Parra, José Carlos; de la Garza, Jorge; 2018, Vol. 34 Issue 147, p139-148. 10p.)

Por otro lado, siempre se reconoce que existen limitaciones o problemas de comportamiento organizacional en toda institución u organización; sin embargo, un mejor comportamiento organizacional no garantiza que se mejore el desempleo, ni tampoco soslayará nuestras deficiencias. No puede ser el sustituto de la falta de planeación, la organización inepta, o los controles inadecuados. Las desviaciones en el comportamiento suelen confundirse y dañar a los empleados tanto como a la organización. Tanto los trabajadores como los gerentes pueden perjudicar a otros empleados con demasiado interés y cuidado. Uno de los principales problemas que ha tenido el comportamiento 
organizacional ha sido la tendencia de las empresas a querer tener resultados inmediatos cuantitativos de los programas de comportamiento.

Esto algunas veces lleva a los gerentes a enrolarse en la nueva moda, y a preocuparse por los síntomas mientras descuidan los problemas subyacentes, o a fragmentar sus esfuerzos dentro de la organización. Los programas de desarrollo organizacional enfocado a un cambio sistemático y a la generación de planes estratégicos a largo plazo para la administración de recursos humanos han ayudado a promover las expectativas realistas que consideran al empleado como una entidad productiva. Otro reto que afronta el comportamiento organizacional es analizar si las ideas que ha sido generadas y aprobadas durante el tiempo de crecimiento organizacional y amplios recursos económicos caminarán con igual éxito en nuevas situaciones ¿puede el comportamiento organizacional adaptar al cambio? Otra preocupación constante respecto al comportamiento organizacional es que sus conocimientos y técnicas pueden utilizarse para manipular a la persona tanto como para ayudarla a desarrollar su potencial.

Las personas que no tiene respeto por la dignidad podrían aprender los conceptos del comportamiento organizacional y utilizarlos para sí mismos con fines egoístas. La filosofía del comportamiento organizacional tiene un enfoque de apoyo y está orientada a los recursos humanos. Busca mejorar el ambiente humano y ayudar a las personas a que desarrollen su propio potencial. Sin embargo, los conocimientos y técnicas de esta teoría pueden utilizarse para obtener resultados positivos y negativos. Esta posibilidad existe en cualquier campo de conocimiento, por lo que no implica una especial limitación, no obstante, debemos ser cautos y estar conscientes de lo que sabemos del ser humano no lo utilizaremos para manipularlo. Esto quiere decir que las autoridades de la organización deben estar a cargo de personas con alta integridad ética y moral.

Además, en la administración de recursos humanos no existen leyes, es decir, es contingente ya que en la manera que ésta se desarrolle depende de la situación organizacional, del amiente, de la tecnología empleada, de la concepción que se tenga en la institución acerca del hombre y de la naturaleza, de la educación, de la gestión, etc. A medida que estos factores cambian, cambia la manera de administrar los recursos humanos. El personal tiene el poder de dirigir el curso de las instituciones y depende de ellas directamente que se pueda reflejar el buen funcionamiento de las mismas. Para que una institución refleje buena calidad en sus servicios, mediante la participación de todo 
el potencial humano, es necesario que las personas desempeñen su trabajo en un ambiente laboral que lo haga más productivo y eficiente. De este modo, si se desea otorgar satisfacción total a los usuarios u cliente externos, es necesario que se empiece por ofrecer un nivel de satisfacción superior a los clientes o usuarios internos.

Para que esto funcione, es inexcusable que en las unidades de desarrollo de la institución, la forma de organización que cuenta la institución u el comportamiento organizacional en sus diferentes dimensiones, sea analizada desde la percepción de los propios usuarios; puesto que en el servicio educativo, los procesos deben ser eficientes debido al gran número de clientes externos que se tienen, ya que se necesita que todo esté perfectamente sincronizado entre las diferentes comisiones y el personal, para llevar a cabo las funciones en el tiempo establecido.

Por lo mencionado la investigación formuló como problema: ¿Cómo es el comportamiento organizacional en la gestión educativa?; y como objetivo: Caracterizar el comportamiento organizacional en la mejora de la gestión educativa.

\section{ESTRATEGIAS METODOLÓGICAS o MATERIALES Y MÉTODOS}

La investigación fue básica de diseño descriptivo con revisión sistemática, las revisiones sistemáticas son investigaciones científicas en las cuales la unidad de análisis son los estudios originales primarios, constituyen una herramienta esencial para sintetizar la información científica disponible, incrementar la validez de las conclusiones de estudios individuales e identificar áreas de incertidumbre donde sea necesario realizar investigación (Hernández, 2016, p.217). Es descriptiva, porque se recogió información relacionada al comportamiento organizacional en la mejora de la gestión educativa (Tamayo y Tamayo, 2009, p. 54). Por lo mencionado, se revisó información científica y se visualizó como solucionar una necesidad en la organización de la entidad pública, para mejorar la gestión organizacional en la ejecución presupuestal municipal y otras deficiencias existentes.

Los procedimiento de recolección de datos se realizó a través de una tabla de registro de datos y la fuente fue revisión bibliográfica de artículos de investigaciones tanto nacionales como internacionales que tuvieron como tema principal el comportamiento organizacional en la gestión educativa; de todos los artículos que se encontraron, se incluyeron los estudios (10) más importantes según nivel de evidencia y se excluyeron 
los menos relevantes. Se estableció la búsqueda siempre y cuando se tuvo acceso al texto completo del artículo científico.

La técnica de análisis utilizada fue a través del análisis de la revisión sistemática evaluando cada uno de los artículos para una comparación de los puntos o características en las cuales concuerda y los puntos en los que existe discrepancia entre artículos nacionales e internacionales. Además, de acuerdo a criterios técnicos pre establecidos, se realizó una evaluación crítica e intensiva de cada artículo, a partir de ello, se determinó la calidad de la evidencia y la fuerza de recomendación para cada artículo. 


\section{RESULTADOS Y DISCUSIÓN: RESULTADOS}

Tabla 1: Estudios revisados sobre el comportamiento organizacional en la gestión educativa 2020 DATOS DE LA PUBLICACIÓN

\begin{tabular}{|c|c|c|c|c|c|c|c|}
\hline \multicolumn{2}{|c|}{ 1. Autor } & Año & \multicolumn{3}{|c|}{ Nombre de la Investigación } & $\begin{array}{c}\text { Revista donde se ubica la } \\
\text { Publicación }\end{array}$ & $\begin{array}{l}\text { Volumen y } \\
\text { número }\end{array}$ \\
\hline \multicolumn{2}{|c|}{$\begin{array}{lr}\text { Trigos Carrillo, Lina } & \text { Marcela } \\
\text { Carreño, Claudia } & \text { Inés } \\
\text { García, Clara } & \text { Inés } \\
\text { Álvarez, Inéride } & \end{array}$} & 2017 & \multicolumn{3}{|c|}{$\begin{array}{l}\text { Innovación y prácticas pedagógicas en la } \\
\text { educación superior: perspectivas teóricas, } \\
\text { investigación y experiencias }\end{array}$} & $\begin{array}{c}\text { Colombia. } \\
\text { http://eds.b.ebscohost. } \\
\text { com/eds/detail/detail?vid=5\&sid } \\
=44 d 8 \mathrm{a} 4 \mathrm{cb}-264 \mathrm{c}-491 \mathrm{c}-8 \mathrm{c} 59- \\
\text { fd484a62a39d\%40pdc-v- } \\
\text { essmgr06\&bdata=Jmxhbmc9ZX } \\
\text { MtbG12ZQ\%3d\%3d\#AN } \\
=1813908 \& \mathrm{db}=\text { nlebk }\end{array}$ & $\begin{array}{l}\text { Editorial } \\
\text { Universidad del } \\
\text { Rosario, 2017. }\end{array}$ \\
\hline \multicolumn{8}{|c|}{ CONTENIDO DE LA PUBLICACIÓN } \\
\hline $\begin{array}{c}\text { Tipo y Diseño } \\
\text { de } \\
\text { Investigación }\end{array}$ & $\begin{array}{l}\text { Población y } \\
\text { Muestra }\end{array}$ & Instrumentos & Aspectos ético & Resultado & & Conclusión & \\
\hline $\begin{array}{l}\text { Enfoque } \\
\text { mixto, } \\
\text { cualitativo- } \\
\text { cuantitativo. }\end{array}$ & $\begin{array}{l}23 \\
\text { administrador } \\
\text { es, } 44 \\
\text { profesores, } \\
478 \\
\text { estudiantes, } 6 \\
\text { instituciones } \\
\text { educativas. } \\
\text { Mexico } \\
\text { (3),Colombia } \\
\text { (2), Perú (1) }\end{array}$ & Cuestionario & $\begin{array}{l}\text { Código de } \\
\text { ética en la } \\
\text { investigación }\end{array}$ & $\begin{array}{l}\text { Existe un } \\
\text { proceso de } \\
\text { innovación } \\
\text { institucional, } \\
\text { asimismo se } \\
\text { cuenta con } \\
\text { personal } \\
\text { especializado } \\
\text { en la gestión } \\
\text { del } \\
\text { conocimiento }\end{array}$ & $\begin{array}{l}\text { En } \\
\text { clar } \\
\text { edu } \\
\text { ges } \\
\text { cab } \\
\text { pro } \\
\text { un } \\
\text { inc } \\
\text { Un } \\
\text { en } \\
\text { estı } \\
\text { cuá }\end{array}$ & $\begin{array}{l}\text { esta investigación, no es posible de } \\
\text { a entre la gestión administrati } \\
\text { cativa; los resultados sugieren que, } \\
\text { ión administrativa orientadas a la } \\
\text { en las instituciones educativas } \\
\text { esores y administradores, no neces } \\
\text { mejor nivel de prácticas de inn } \\
\text { rporación de la tecnología en opini } \\
\text { posible explicación a este fenóme } \\
\text { la percepción entre administra } \\
\text { diantes sobre qué es innovación ec } \\
\text { es el propósito de la innovación ed }\end{array}$ & $\begin{array}{l}\text { rminar una relación } \\
\text { y la innovación } \\
\text { bien las prácticas de } \\
\text { inovación se llevan } \\
\text { en la opinión de } \\
\text { iamente conducen a } \\
\text { vación educativa e } \\
\text { n de los estudiantes. } \\
\text { o sería la diferencia } \\
\text { ores, profesores y } \\
\text { cativa, y sobre todo } \\
\text { cativa. }\end{array}$ \\
\hline
\end{tabular}




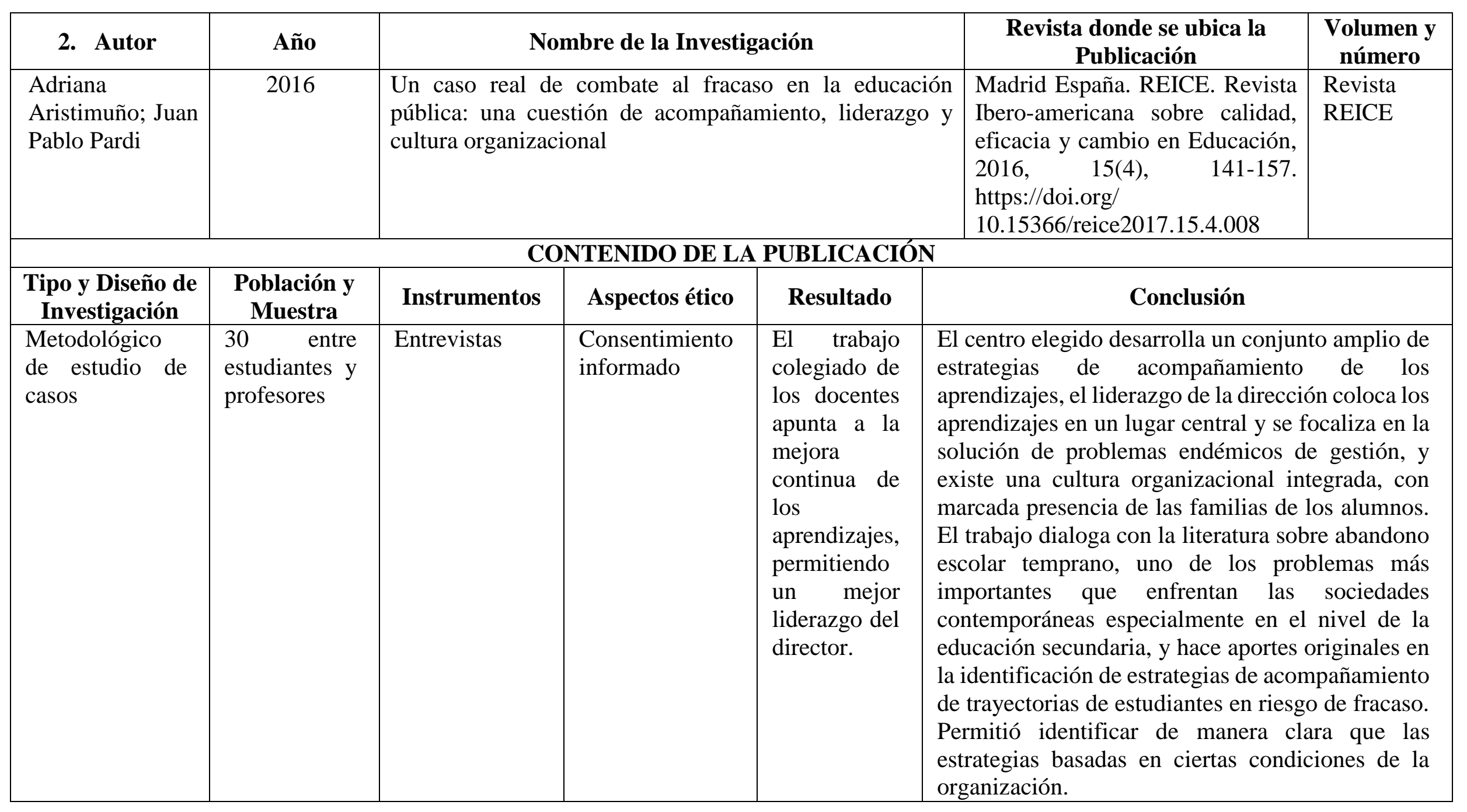




\begin{tabular}{|c|c|c|c|c|c|c|}
\hline 3. Autor & Año & \multicolumn{3}{|c|}{ Nombre de la Investigación } & $\begin{array}{c}\text { Revista donde se } \\
\text { ubica la } \\
\text { Publicación }\end{array}$ & $\begin{array}{l}\text { Volumen y } \\
\text { número }\end{array}$ \\
\hline $\begin{array}{l}\text { Marcia } \\
\text { Sierdvski, }\end{array}$ & 2020 & \multicolumn{3}{|c|}{$\begin{array}{l}\text { Relación de la estrategia como práctica social para la } \\
\text { construcción de indicadores en el pilar social de la sostenibilidad } \\
\text { organizacional. }\end{array}$} & $\begin{array}{l}\text { Argentina } \\
\text { REUNIR revista } \\
\text { de } \\
\text { Administración, } \\
\text { ciencias contables } \\
\text { y sustentable }\end{array}$ & $\begin{array}{l}\text { Vol. 10, } \\
\text { Número1. }\end{array}$ \\
\hline \multicolumn{7}{|c|}{ CONTENIDO DE LA PUBLICACIÓN } \\
\hline $\begin{array}{c}\text { Tipo y } \\
\text { Diseño de } \\
\text { Investigación }\end{array}$ & $\begin{array}{c}\text { Población y } \\
\text { Muestra }\end{array}$ & Instrumentos & Aspectos ético & Resultado & \multicolumn{2}{|c|}{ Conclusión } \\
\hline $\begin{array}{l}\text { Artículos } \\
\text { científicos } \\
\text { enfoque } \\
\text { cualitativo }\end{array}$ & 150 empresas & Entrevista & $\begin{array}{l}\text { Consentimiento } \\
\text { informado }\end{array}$ & \begin{tabular}{lrr} 
La & \multicolumn{2}{c}{ investigación } \\
cualitativa & pudo \\
comprender & & los \\
sentimientos, & & las \\
aspiraciones & y & las \\
expectativas & de & los \\
empleados, y & así \\
identificar & los \\
problemas importantes \\
para los empleados y \\
definir & como \\
construcciones & de \\
análisis del pilar social.
\end{tabular} & $\begin{array}{l}\text { Las organizaciones } \\
\text { sus estrategias } \\
\text { sostenibilidad } \\
\text { decisiones basa } \\
\text { responsabilidad } \\
\text { corporativa, cons } \\
\text { prácticas dentro c } \\
\text { organizacional, } \\
\text { mejorar su calidad } \\
\text { trabajadores en } \\
\text { empresas. }\end{array}$ & $\begin{array}{l}\text { deben definir } \\
\text { sadas en la } \\
\text { ial, y rus } \\
\text { as en la } \\
\text { social } r y \\
\text { derando sus } \\
\text { su contexto } \\
\text { permitiendo } \\
\text { de vida de los } \\
\text { las mejores }\end{array}$ \\
\hline
\end{tabular}




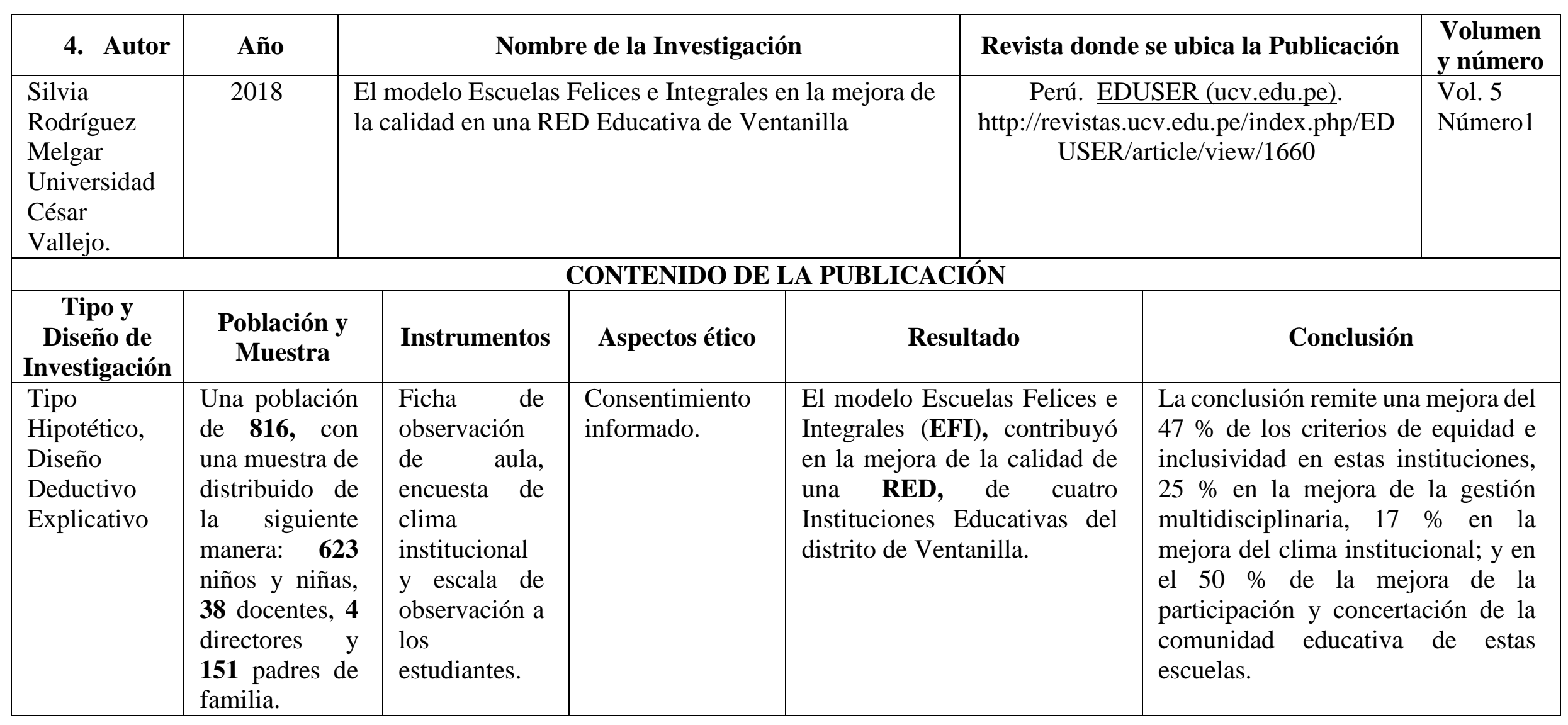




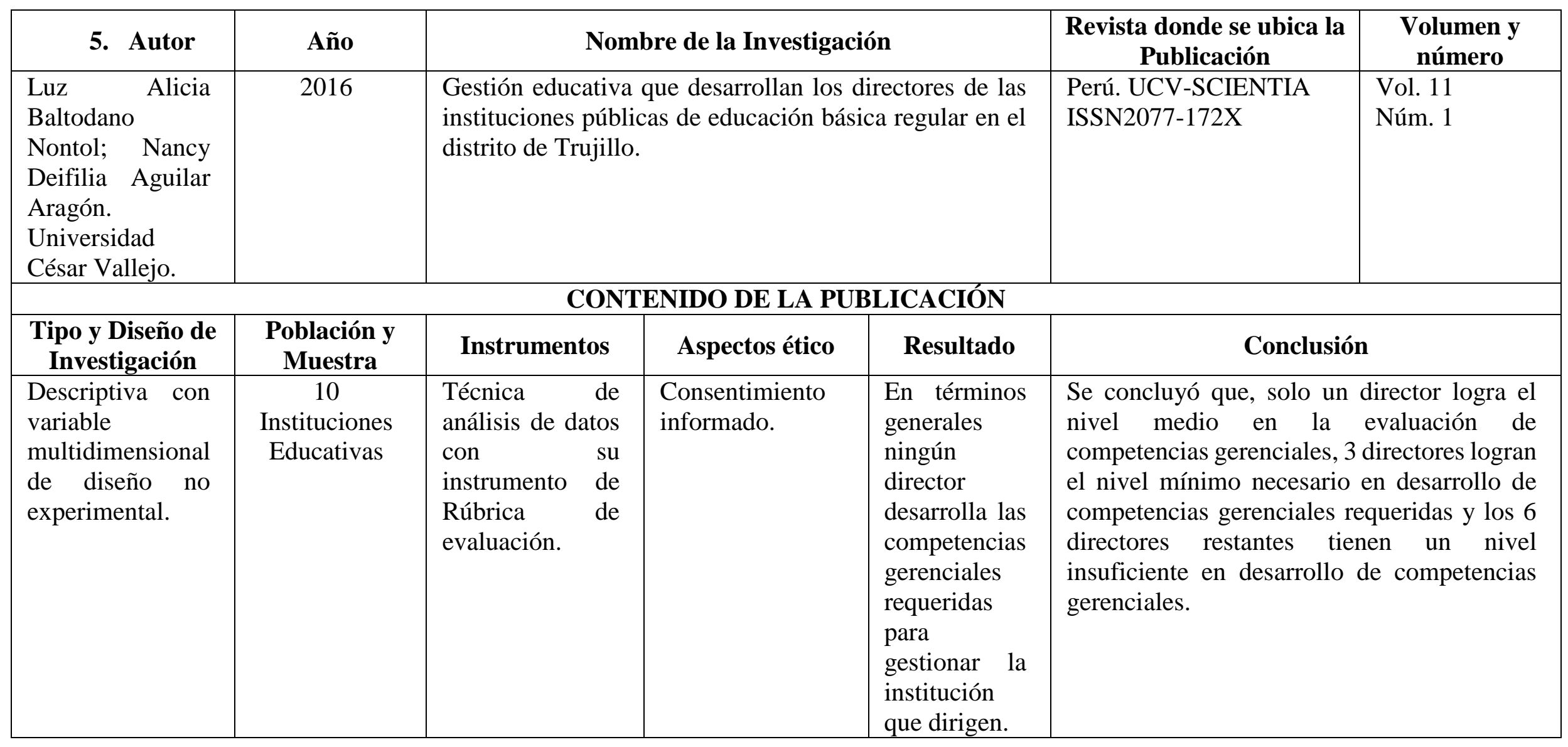




\begin{tabular}{|c|c|c|c|c|c|c|}
\hline 6. Autor & Año & \multicolumn{3}{|c|}{ Nombre de la Investigación } & $\begin{array}{l}\text { Revista donde se } \\
\text { ubica la } \\
\text { Publicación }\end{array}$ & $\begin{array}{l}\text { Volumen y } \\
\text { número }\end{array}$ \\
\hline $\begin{array}{l}\text { Guiselle M. } \\
\text { Garbanzo- } \\
\text { Vargas }\end{array}$ & 2016 & \multicolumn{3}{|c|}{$\begin{array}{l}\text { Desarrollo organizacional y los procesos de cambio en las } \\
\text { instituciones educativas, un reto de la gestión de la educación. }\end{array}$} & $\begin{array}{ll}\text { Revista } & \\
\text { Educación } & - \\
\text { Universidad de } \\
\text { Costa Rica } \\
\text { Costa Rica. }\end{array}$ & $\begin{array}{l}\text { Vol. } 40 \\
\text { Núm. } 1 \\
\text { Enero-junio, } \\
2016, \\
\text { pp. } 67-87 .\end{array}$ \\
\hline \multicolumn{7}{|c|}{ CONTENIDO DE LA PUBLICACIÓN } \\
\hline $\begin{array}{l}\text { Tipo y Diseño } \\
\text { de } \\
\text { Investigación }\end{array}$ & $\begin{array}{l}\text { Población y } \\
\text { Muestra }\end{array}$ & Instrumentos & Aspectos ético & Resultado & \multicolumn{2}{|c|}{ Conclusión } \\
\hline $\begin{array}{l}\text { Artículos } \\
\text { científicos } \\
\text { enfoque } \\
\text { cualitativo }\end{array}$ & & Entrevista & $\begin{array}{l}\text { Consentimiento } \\
\text { informado. }\end{array}$ & $\begin{array}{l}\text { Las organizaciones } \\
\text { educativas que aprenden en } \\
\text { sí mismas requieren, desde } \\
\text { su gestión, considerar } \\
\text { distintos elementos que, } \\
\text { articulados, propician la } \\
\text { eficacia organizacional con } \\
\text { base en el aprendizaje mutuo } \\
\text { como organizaciones } \\
\text { inteligentes. }\end{array}$ & \multicolumn{2}{|c|}{$\begin{array}{l}\text { Se concluye que, el liderazgo } \\
\text { compartido mediante una eficiente } \\
\text { gestión es la clave en las organizaciones } \\
\text { que aprenden, la responsabilidad, la } \\
\text { dirección de los procesos y el } \\
\text { cumplimiento de los objetivos que } \\
\text { comparten dentro de la organización de } \\
\text { manera general y el trabajo debe ser con } \\
\text { un enfoque sistémico, dado que la } \\
\text { totalidad de sus miembros se identifican } \\
\text { con la institución. }\end{array}$} \\
\hline
\end{tabular}




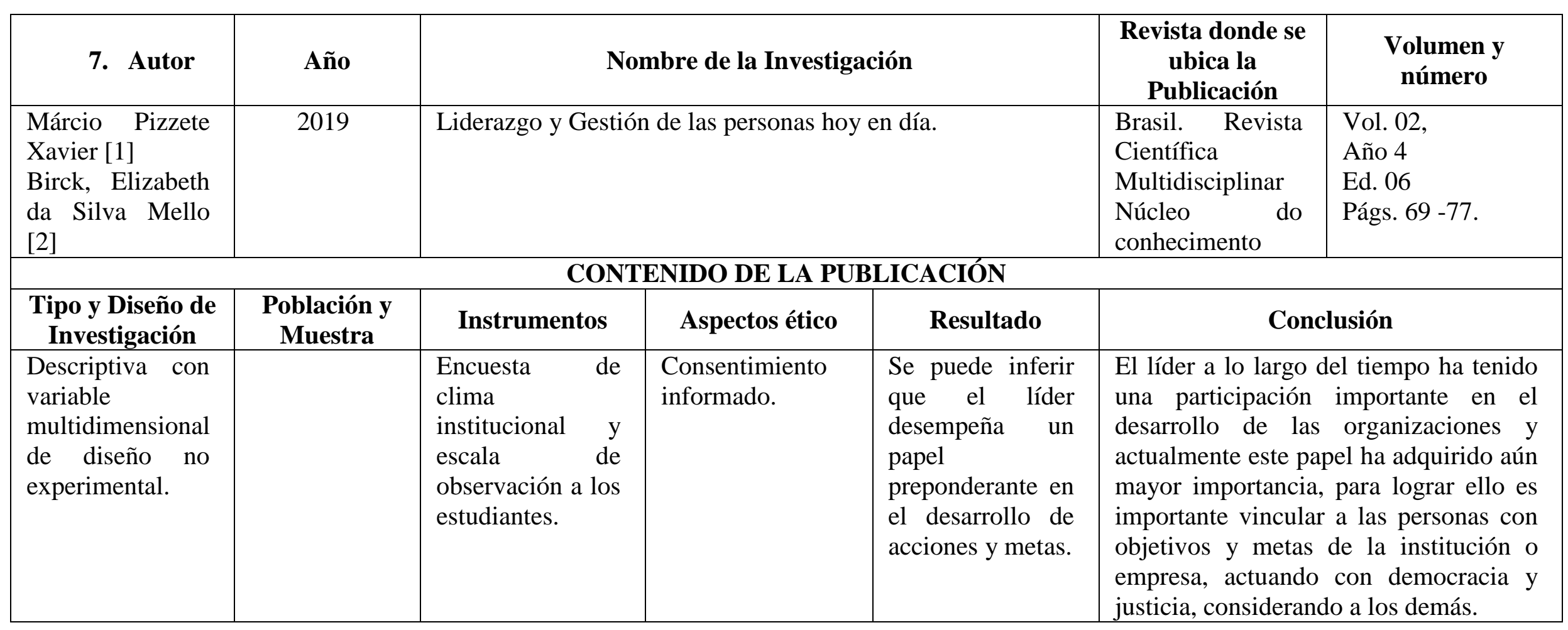




\begin{tabular}{|c|c|c|c|c|c|c|}
\hline 8. Autor & Año & \multicolumn{3}{|c|}{ Nombre de la Investigación } & $\begin{array}{l}\text { Revista donde se } \\
\text { ubica la Publicación }\end{array}$ & $\begin{array}{c}\text { Volumen } \\
\mathbf{y} \\
\text { número }\end{array}$ \\
\hline $\begin{array}{l}\text { MSc. Jorge Zambrano } \\
\text { Campoverde [1] } \\
\text { MSc. Miguel Angel Ramón } \\
\text { Pineda [1] } \\
\text { Dr.C.Enrique Espinoza-Freire } \\
\text { [1] }\end{array}$ & 2017 & \multicolumn{3}{|c|}{$\begin{array}{l}\text { Estudio sobre el clima organizacional en docentes de la } \\
\text { universidad técnica de Machala. }\end{array}$} & $\begin{array}{l}\text { Cuba. Universidad y } \\
\text { Sociedad/Universidad } \\
\text { de Cienfuegos. }\end{array}$ & $\begin{array}{l}\text { Vol. } 9 \\
\text { Núm. } 2\end{array}$ \\
\hline \multicolumn{7}{|c|}{ CONTENIDO DE LA PUBLICACIÓN } \\
\hline Tipo y Diseño de Investigación & $\begin{array}{c}\text { Población } \\
\text { y } \\
\text { Muestra }\end{array}$ & Instrumentos & Aspectos ético & Resultado & \multicolumn{2}{|l|}{ Conclusión } \\
\hline $\begin{array}{l}\text { Descriptiva con variable } \\
\text { multidimensional de diseño no } \\
\text { experimental. }\end{array}$ & $\begin{array}{l}175 \\
\text { docentes }\end{array}$ & $\begin{array}{l}\text { Encuesta } \\
\text { con } \\
\text { preguntas } \\
\text { cerradas. }\end{array}$ & $\begin{array}{l}\text { Consentimiento } \\
\text { informado. }\end{array}$ & $\begin{array}{l}\text { El clima organizacional } \\
\text { en universidades se centra } \\
\text { en la satisfacción laboral, } \\
\text { éxitor académico, } \\
\text { construcción re } \\
\text { significados, que hacer del } \\
\text { docente y calidad } \\
\text { educativa. }\end{array}$ & \multicolumn{2}{|c|}{$\begin{array}{l}\text { El clima organizacional ocupa un } \\
\text { espacio relevante en la gestión de } \\
\text { las organizaciones y estudiarlo se } \\
\text { torna una medición empírica para } \\
\text { obtener un alto desempeño } \\
\text { laboral, eleva el compromiso y la } \\
\text { cooperación entre sujetos para que } \\
\text { se sienten parte de un grupo de } \\
\text { trabajo satisfactorio. }\end{array}$} \\
\hline
\end{tabular}




\begin{tabular}{|c|c|c|c|c|c|c|}
\hline 9. Autor & Año & \multicolumn{3}{|c|}{ Nombre de la Investigación } & $\begin{array}{c}\text { Revista donde se } \\
\text { ubica la } \\
\text { Publicación }\end{array}$ & Volumen y número \\
\hline $\begin{array}{l}\text { Columba } \\
\text { consuelo- } \\
\text { Bravo[I] } \\
\text { Ileana } \\
\text { Sarmentero- } \\
\text { Bom[II] } \\
\text { Olga Gómez- } \\
\text { Figueroa[II] } \\
\text { Odalys } \\
\text { Falcon[II] }\end{array}$ & 2018 & $\begin{array}{l}\text { Procedimiento } p \\
\text { organizacional }\end{array}$ & el estudio & comportamiento & $\begin{array}{l}\text { Ingeniería } \\
\text { Industrial }-\mathrm{La} \\
\text { Habana. }\end{array}$ & $\begin{array}{l}\text { Vol. } 39 \\
\text { Núm. } 1\end{array}$ \\
\hline \multicolumn{7}{|c|}{ CONTENIDO DE LA PUBLICACIÓN } \\
\hline $\begin{array}{l}\text { Tipo y Diseño de } \\
\text { Investigación }\end{array}$ & $\begin{array}{l}\text { Población y } \\
\text { Muestra }\end{array}$ & Instrumentos & Aspectos ético & Resultado & \multicolumn{2}{|c|}{ Conclusión } \\
\hline $\begin{array}{ll}\text { Métodos } & \\
\text { teóricos } & \text { y } \\
\text { empíricos: } & \\
\text { análisis } & \text { y } \\
\text { síntesis, } & \\
\text { inducción } & \text { y } \\
\text { deducción. } & \end{array}$ & MYPES & $\begin{array}{l}\text { Lista de chequeo, } \\
\text { encuesta, } \\
\text { entrevista. }\end{array}$ & $\begin{array}{l}\text { Consentimiento } \\
\text { informado. }\end{array}$ & $\begin{array}{l}\text { Las variables que } \\
\text { con mayor } \\
\text { frecuencia se } \\
\text { encontraron } \\
\text { fueron: } \\
\text { individual, grupal } \\
\text { y estructura } \\
\text { (sistema } \\
\text { organizativo.) }\end{array}$ & $\begin{array}{l}\text { La aplicación del p1 } \\
\text { conocer las cualida } \\
\text { comercializadoras } \\
\text { variables del CO q } \\
\text { empresas y los cor } \\
\text { vinculación de } \\
\text { esenciales para pod } \\
\text { faciliten la gestión }\end{array}$ & $\begin{array}{l}\text { cedimiento seguido permitió } \\
\text { les esenciales de las PYME } \\
\text { de productos lácteos, las } \\
\text { resultan esenciales en estas } \\
\text { lomerados resultantes de la } \\
\text { s mismas, siendo estos } \\
\text { r determinar las acciones que } \\
\text { xitosa de dichas empresas. }\end{array}$ \\
\hline
\end{tabular}




\begin{tabular}{|l|c|l|l|l|}
\hline \multicolumn{1}{|c|}{ 10. Autor } & Año & Nombre de la Investigación & \multicolumn{1}{c|}{$\begin{array}{c}\text { Revista donde se ubica la Publicación } \\
\text { número }\end{array}$} \\
\hline $\begin{array}{l}\text { Diego Andrés Rivera } \\
\text { Porras [1] Milena } \\
\begin{array}{l}\text { Sandra } \\
\text { Carrillo Sierra [2] }\end{array}\end{array}$ & 2018 & $\begin{array}{l}\text { Cultura Organizacional, retos y } \\
\text { desafíos para las organizaciones } \\
\text { saludables }\end{array}$ & $\begin{array}{l}\text { Venezuela. } \\
\text { Espacios. } \\
\text { https://www.revistaespacios.com/a18v39 } \\
\text { n22/a18v39n22p27.pdf }\end{array}$ \\
$\begin{array}{l}\text { Forgiony Santos [1] } \\
\begin{array}{l}\text { Irma Lizeth Nuván } \\
\text { Hurtado [4] }\end{array}\end{array}$ \\
$\begin{array}{l}\text { Astrid Carolina Rozo } \\
\text { Sánchez [5] }\end{array}$
\end{tabular}




\section{DISCUSIÓN}

Posterior a la revisión sistemática de los artículos, del 100\%, el 90\% corresponden a América Latina y el Caribe, siendo Perú el que más resalta con el 20\%, seguido de Colombia, Argentina, Costa rica, Brasil, Cuba y Venezuela. Sin embargo, el 10\% corresponde a Europa al país España. En relación a los diseños y tipos de estudios el 100\% son descriptivos con diferentes variantes como cualitativo, revisión, explicativo, cuantitativo.

En función al análisis de las variables comportamiento organizacional de la gestión educativa tenemos a Trigos, Carreño, García, Alvarez, quienes concluyen que, gestión administrativa y la innovación educativa; los resultados sugieren que, si bien las prácticas de gestión administrativa orientadas a la innovación se llevan cabo en las instituciones educativas. Por su parte Aristimuño y Pardi, establecen que la organización responde a la mejora continua de los aprendizajes. Sin embargo, para Sierdvski, comprobó que los sentimientos, las aspiraciones y las expectativas de los empleados forman parte de la organización en la gestión educativa, por ello la sostenibilidad social, la responsabilidad social y corporativa forman parte del contexto organizacional mejorando la calidad de vida de los colaboradores. Igualmente, Silva, resalta las escuelas felices e integrales contribuyeron a la mejora de la calidad, fortaleciendo la organización en la gestión educativa.

También Baltodano, Aguilar, determinaron que ningún director desarrolla las competencias gerenciales requeridas para gestionar la institución que dirigen, necesitando fortalecer competencias gerenciales. Garbanzo-Vargas concluye que el liderazgo compartido mediante una eficiente gestión es la clave en la organización, generando mayor producción y por ende mejor formación. Asimismo, Prizzete, Da Silva, señala que el líder a tenido una participación importante en el desarrollo de las organizaciones siendo necesario vincular con los objetivos. Igualmente, Zambrano, Ramón, Espinoza-Freire, determinaron que el clima organizacional ocupa un espacio en la gestión de organizaciones centrado en la satisfacción laboral, éxito académico, quehacer del docente y calidad educativa. Para Rivera, Carrillo, Forgiony, Nuván y Rozo, determinó que, la cultura organizacional percibida como necesidad en la creación de organizaciones educativas saludables amerita cambios sociales, económicos, cultural, prácticas, salid, seguridad. Por último, Bravo, Bom, Gómez-Figueroa y Falcón, los procedimientos en la organización permitió conocer cualidades esenciales de las MYPES. 


\section{CONCLUSIONES O CONSIDERACIONES FINALES}

De los 10 artículos científicos, sobre el comportamiento organizacional en la gestión educativa en el contexto de la crisis económica internacional, corresponden a diferentes bases de datos, todos ellos al tipo y diseño de estudios descriptivos.

El comportamiento organizacional asegura un mejor desarrollo de la gestión educativa, la organización facilita procesos de desarrollo institucional que responde a los objetivos estratégicos.

El comportamiento organizacional en la gestión educativa es deficiente dado que los usuarios internos y externos no se sienten satisfechos con el servicio brindado por parte los trabajadores direccionados por el líder de las Instituciones Educativas.

Existe un proceso de innovación institucional para fortalecer la gestión intelectual asimismo se cuenta con personal especializado en la gestión del conocimiento.

\section{LISTA DE REFERENCIAS}

Gnanarajan; Ariyaratnam; Kengatharan; Navaneethakrishnan; Velnampy, Thirunavukkarasu, (2014).

Munir; Yasin; Shabbir; Ali; Tariq; Chani; Orangzab, Muhammad; Abbas, Mazhar; (2019) Special Issue, Vol. 6, p1-21, 21p.

Martín; Leiva; Brenes; (2019), Vol. 64 Issue 2, p1-18. 18p.

Chiavenato, (2007), ... bem como nas especificidades. Revista Brasileira de Pesquisa em Turismo. São Paulo, 9(3), pp. 422-441, set./dez. 2015 p. 441. Disponible en:

https://scholar.google.com.pe/scholar?q=Chiavenato, $+(2007),+$ p.+441.\&hl=es\&as _s $\underline{\mathrm{dt}=0 \& \text { as_vis }=1 \& \text { oi }=\text { scholart. }}$

Arredondo Trapero, Florina; Vázquez Parra, José Carlos; de la Garza, Jorge; (2018), Vol.

34 Issue 147, p139-148. 10p.

Hernández, M. (2016). Influencias suizas en la educación española e iberoamericana.Disponible:https://scholar.google.es/citations?user=Xhq9V2gAAA AJ\&hl=es\#d=gs_md_citad\&u=\%2Fcitations\%3Fview_op\%3Dview_citation\%26hl \%3Des\%26user\%3DXhq9V2gAAAAJ\%26citation_for_view\%3DXhq9V2gAAAA J\%3AClCfbGk0d_YC\%26tzom\%3D300. 
Trigos L, Carreño C, García C, Álvarez, I. (2017). Innovación y prácticas pedagógicas en la educación superior: perspectivas teóricas, investigación y experiencias Colombia. Editorial Universidad del Rosario, 2017. http://eds.b.ebscohost. com/eds/detail/detail?vid=5\&sid=44d8a4cb-264c-491c-8c59fd484a62a39d\%40pdc-vessmgr06\&bdata=Jmxhbmc9ZXMtbG12ZQ\%3d\%3d\#AN=1813908\&db=nlebk

Aristimuño A, Pardi J. (2016). Un caso real de combate al fracaso en la educación pública: una cuestión de acompañamiento, liderazgo y cultura organizacional. Madrid España. REICE. Revista Ibero-americana sobre calidad, eficacia y cambio en Educación, 2016, 15(4), 141-157. Revista REICE. https://doi.org/10.15366/reice2017.15.4.008

Sierdvski, M. (2020). Relación de la estrategia como práctica social para la construcción de indicadores en el pilar social de la sostenibilidad organizacional. Argentina REUNIR revista de Administración, ciencias contables y sustentable Vol. 10, Número1.

Rodríguez S. (2018) El modelo Escuelas Felices e Integrales en la mejora de la calidad en una RED Educativa de Ventanilla Perú. EDUSER Vol. 5. Número1. (ucv.edu.pe). http://revistas.ucv.edu.pe/index.php/EDUSER/article/view/1660

Baltodano L, Aguilar N. (2016). Gestión educativa que desarrollan los directores de las instituciones públicas de educación básica regular en el distrito de Trujillo.Perú. UCV-SCIENTIA ISSN2077-172X Vol. 11. Núm. 1. https://dialnet.unirioja.es/descarga/articulo/7304386.pdf

Garbanzo-Vargas, G. (2016). Desarrollo organizacional y los procesos de cambio en las instituciones educativas, un reto de la gestión de la educación. Revista Educación - Universidad de Costa Rica - Costa Rica. Vol. 40. Núm. 1. Enero-junio, 2016. p. 67-87.

https://www.researchgate.net/publication/289569269_Desarrollo_organizacional_y los_procesos_de_cambio_en_las_instituciones_educativas_un_reto_de_la_gestion_ de_la_educacion 
Márcio X, Da Silva B. (2019). Liderazgo y Gestión de las personas hoy en día. Brasil. Revista Científica Multidisciplinar Núcleo do conhecimento. Vol. 02. Año 4. Ed. 06. p 69 -77. https://www.nucleodoconhecimento.com.br/administracion-deempresas/liderazgo-y-gestion

Zambrano J, Ramón M, Espinoza-Freire E. (2017). Estudio sobre el clima organizacional en docentes de la universidad técnica de Machala.Cuba. Universidad y Sociedad/Universidad de Cienfuegos. Vol. 9. Núm. 2. http://scielo.sld.cu/scielo.php?script=sci_arttext\&pid=S2218-36202017000200022

Rivera D, Carrillo S, Forgiony J, Nuván I, Rozo A. (2018). Cultura Organizacional, retos y desafíos para las organizaciones saludables. Venezuela. Espacios. Vol. 39. Núm. 22. https://www.revistaespacios.com/a18v39n22/a18v39n22p27.pdf

Consuelo-Bravo C, Sarmentero-Bom I, Gómez-Figueroa O, Falcon O. (2018). Procedimiento para el estudio del comportamiento organizacional Ingeniería Industrial - La Habana.Vol. 39. Núm. 1. http://scielo.sld.cu/scielo.php?script=sci_arttext\&pid=S1815-59362018000100010 